\title{
Bruhat graphs and pattern avoidance
}

\author{
Christopher Conklin and Alexander Woo
}

\begin{abstract}
We characterize permutations whose Bruhat graphs can be drawn in the plane and those whose Bruhat graphs can be drawn on the torus. In particular, we show these properties are characterized by avoiding finitely many permutations.
\end{abstract}

AMS 2000 SUBJECT CLASSIFICATIONS: Primary 05C25; secondary 05A05, $05 \mathrm{E} 15$.

KEYWORDS AND PHRASES: Bruhat graph, pattern avoidance, planar graph.

\section{Introduction}

The set of all permutations (of an arbitrary finite number of elements) admits a partial order known as pattern containment order. This partial order is known to admit infinite antichains [23, 20]. On the other hand, in almost all cases where the set of permutations satisfying some property has been characterized by pattern containment, the number of permutations involved is finite. For some properties, such as in $[25,9,6,7]$, the number of permutations is moderate to quite large, so this phenomenon seems to involve more than merely the natural inclination of mathematicians to study simpler examples. Our goal in this paper is to begin the exploration of one possible explanation for this finiteness.

Associated to each permutation is a directed graph known as the Bruhat graph. (For definitions see Section 2.) Whenever a permutation $\pi$ is contained in a permutation $\sigma$ (so $\pi \leq \sigma$ in pattern containment order), the Bruhat graph of $\pi$ is a subgraph of the Bruhat graph of $\sigma$. While not all properties characterized by pattern containment can be reduced to properties of Bruhat graphs, many of the properties that have been so characterized, especially those coming from algebraic geometry or representation theory, have a graph theoretic description. For example, the permutations $w$ avoiding 3412 and 4231 are exactly the ones whose Bruhat graphs are regular, meaning that they have the same number of edges at each vertex; these are also the ones associated with smooth Schubert varieties [19, 13].

arXiv: 1107.4812 
In this paper we study the permutations whose Bruhat graphs can be drawn (without crossings) on the plane or on the torus. We show that the planar permutations are precisely the ones that avoid 321 and have length at most 3, and their Bruhat graphs are either a single point, a single edge, (the edge graph of) a square, or a cube. We give an analogous characterization of permutations whose Bruhat graphs can be drawn on the torus; the additional possible graphs are $K_{3,3}$ (the complete bipartite graph on two sets of three vertices) and the 4-cube. By an old theorem of Atkinson [1], our characterizations imply that these properties are characterized by avoidance of finitely many patterns. A computer calculation shows that 29 patterns are needed to characterize planar permutations, and 92 are needed to characterize toroidal ones.

The motivation for considering Bruhat graphs is the Graph Minor Theorem, a deep and surprising result of Robertson and Seymour [22] stating that graph minor order, the partial order on graphs generated by deleting and contracting edges, has no infinite antichains. In particular, the graphs satisfying any property which is preserved under deletion and contraction are characterized by avoiding finitely many graphs. An important special case of the Graph Minor Theorem is the one involving the class of graphs which can be drawn without crossings on a surface of genus $g$ [21], and the original inspiration for graph minor theory was Kuratowski's Theorem, which characterizes graphs that cannot be drawn in the plane. Our results in this paper can therefore be seen as potential first steps towards using graph minor theory to establish either directly or by analogy a possible finiteness result for pattern avoidance under yet unknown hypotheses involving Bruhat graphs.

In particular, we also expect that the set of permutations whose Bruhat graphs can be drawn on a surface of genus $g$ can be characterized by avoidance of a finite number of patterns. However, it appears that any characterization of these permutations, even if not directly in terms of the patterns to be avoided, will be fairly long even for the case $g=2$.

We were particularly inspired by the question of Billey and Weed [9] concerning whether, for a fixed integer $k$, having Kazhdan-Lusztig polynomial $P_{i d, \sigma}(1) \leq k$ is characterized by the permutation $\sigma$ avoiding some finite list of patterns. (Billey and Braden previously showed in [5] that this property is characterized by avoiding a possibly infinite set of patterns.) This Kazhdan-Lusztig polynomial is known to be a property depending only on the Bruhat graph of $\sigma[17,12,15]$, although the aforementioned property is not preserved by deletion and contraction on Bruhat graphs (nor is its negation). 
Our work is in some sense orthogonal to earlier work of Atkinson, Murphy, and Ruskuc $[1,2,3]$ on finitely generated order ideals in pattern containment order. Their work takes the viewpoint that a permutation is a string consisting of distinct integers. Our viewpoint considers permutations as elements of a Coxeter group. Therefore our results are complementary to theirs. Furthermore, pattern avoidance has alternative definitions within the framework of Coxeter groups [8]. We believe it should not be too difficult to extend our work to other Coxeter groups by characterizing the elements with planar or toroidal Bruhat graphs using these Coxeter-theoretic notions of pattern avoidance.

Section 2 is devoted to definitions, while Sections 3 and 4, respectively, give proofs for our theorems about permutations whose Bruhat graphs are planar and toroidal.

\section{Definitions}

We begin with definitions from the combinatorics of Coxeter groups applied to the specific case of the symmetric group $S_{n}$; a standard reference for this material is [10].

By a transposition $t$ we mean some 2-cycle $(i j)$ in the symmetric group. An adjacent transposition is one of the form $(i i+1)$.

Let $\pi \in S_{n}$ be a permutation. The length of $\pi$, denoted $\ell(\pi)$, is the minimum number of adjacent transpositions $s_{i_{1}}, \ldots, s_{i_{\ell}}$ such that $\pi$ can be written as their product $s_{i_{1}} \cdots s_{i_{\ell}}$. An inversion of $\pi$ is a pair of indices $i<j$ with $\pi(i)>\pi(j)$; note that $\ell(\pi)$ is also equal to the number of inversions of $\pi$.

The absolute length of $\pi$, denoted $a(\pi)$, is the minimum number of transpositions $t_{i_{1}}, \ldots, t_{i_{a}}$, not necessarily adjacent transpositions, such that $\pi=t_{i_{1}} \cdots t_{i_{a}}$. If $\pi \in S_{n}$ has $c$ disjoint cycles (counting fixed points as 1cycles), then $a(\pi)=n-c$. By definition, $a(\pi) \leq \ell(\pi)$ for any permutation $\pi$.

Permutations are written in one-line notation unless stated otherwise.

Example 2.1. The permutation $\pi=3412$ has length $\ell(\pi)=4$, and absolute length $a(\pi)=2$.

The symmetric group $S_{n}$ has a partial ordering known as Bruhat order. Define $\pi \prec \sigma$ if $\ell(\pi)<\ell(\sigma)$ and there is a transposition $t$ such that $t \pi=\sigma$ (or equivalently a transposition $t^{\prime}$ such that $\pi t^{\prime}=\sigma$ ). Bruhat order is the transitive closure of $\prec$, so $\pi \leq \tau$ if there exist permutations $\sigma_{1}, \ldots, \sigma_{k} \in S_{n}$ such that $\pi \prec \sigma_{1} \prec \cdots \prec \sigma_{k} \prec \tau$. 


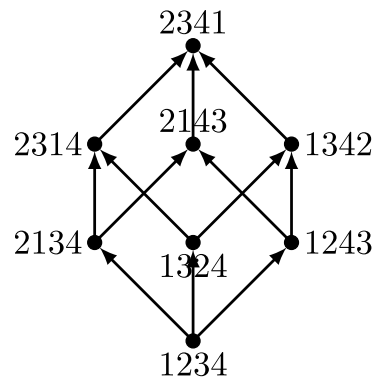

Figure 1: The Bruhat graph $\mathcal{B}(2341)$.

The Bruhat graph for $S_{n}$ is the directed graph whose vertices are the elements of $S_{n}$, with edges defined as follows. Given permutations $\sigma$ and $\tau$, there is an edge $\sigma \rightarrow \tau$ if $\sigma \prec \tau$, meaning that $\ell(\sigma)<\ell(\tau)$ and there exists a transposition $t$ with $\sigma=\tau t$. (Note $\ell(\tau)-\ell(\sigma)$ need not equal 1.) Given a permutation $\pi$, the Bruhat graph for $\pi$, denoted $\mathcal{B}(\pi)$, is the induced subgraph whose vertices are those labeled by permutations $\sigma$ with $\sigma \leq \pi$; this is the largest subgraph with unique sink $\pi$.

Figure 1 shows the Bruhat graph for 2341. For another example, see Figure 2 (later in the paper).

The length of $\pi$ is the length of the longest directed path (necessarily from the identity to $\pi$ ) in $\mathcal{B}(\pi)$, and the absolute length of $\pi$ is the length of the shortest directed path in $\mathcal{B}(\pi)$ from the identity (which is graph theoretically the unique source) to $\pi$ (the unique sink). Sometimes absolute length is defined as the length of the shortest undirected path, but a theorem of Dyer [16] shows these two definitions are equivalent.

Now we give various definitions related to the notion of pattern containment; a standard reference for this subject is [11]. Let $\pi \in S_{k}$ and $\tau \in S_{n}$ with $k \leq n$. We say that $\tau$ (pattern) contains $\pi$ if there exist indices $1 \leq i_{1}<\cdots<i_{k} \leq n$ such that $\tau\left(i_{a}\right)<\tau\left(i_{b}\right)$ if and only if $\pi(a)<\pi(b)$. We say that $\tau$ (pattern) avoids $\pi$ if $\tau$ does not contain $\pi$.

Example 2.2. The permutation 5736241 contains the permutation 3412 in three different ways using the bolded entries: 5736241, 5736241, and 5736241.

In contrast, 135246 avoids 3412 .

We need an analogous notion from graph theory. Given some subset $W$ of the set of vertices of a graph $G$, the subgraph induced by $W$ is the graph containing the vertices in $W$ and every edge of $G$ which connects two 
vertices of $W$. Any subgraph $H \subset G$ that can be formed in this manner (or, in other words, any subgraph $H$ where every edge of $G$ between two vertices of $H$ is also in $H$ ) is called an induced subgraph.

Given this definition, the following proposition is immediate.

Proposition 2.3. If $\tau$ contains $\pi$, then $B(\pi)$ is isomorphic to an induced subgraph of $B(\tau)$ which includes the sink vertex $\tau$.

Proof. Let $i_{1}<\cdots<i_{k}$ be the indices by which $\pi$ is contained in $\tau$. Consider the induced subgraph given by the vertices of $B(\tau)$ labelled by permutations $\sigma$ for which $\sigma(j)=\tau(j)$ for all $j$ not among the containment indices, meaning that $j \neq i_{a}$ for all $a$.

Pattern containment is a partial order relation; the poset of permutations under pattern containment is sometimes called pattern order.

Finally, we need some basic ideas from topological graph theory. A graph is planar if it can be drawn in the plane without edges crossing, and a graph is toroidal if it can be drawn on the surface of a torus without edges crossing. The following theorem is classical and known as Kuratowski's Theorem.

Theorem 2.4. A graph is planar if and only if it contains no subgraph isomorphic to a subdivision of $K_{3,3}$ (the complete bipartite graph with 3 vertices on each side) or $K_{5}$ (the complete graph on 5 vertices).

The precise statement of the analogous theorem for toroidal graphs is not known. The minimal list of graphs to avoid is finite by the Graph Minor Theorem, and a by-no-means exhaustive part of this minimal list with tens of thousands of graphs was computed in [14].

\section{Planar Bruhat graphs}

In this section we give a number of characterizations of permutations with planar Bruhat graphs, beginning with the following.

Theorem 3.1. Let $\sigma$ be a permutation. Then the Bruhat graph $\mathcal{B}(\sigma)$ is planar if and only if $\sigma$ avoids 321 and $\ell(\sigma) \leq 3$.

Proof. The Bruhat graph $\mathcal{B}(321)$ is the complete bipartite graph $K_{3,3}$, which is not planar. Furthermore, $\mathcal{B}(3412)$ contains (as a subgraph) $\mathcal{B}(1432)$ since $1432<3412$ in Bruhat order, and 1432 contains 321 , so $\mathcal{B}(3412)$ is also not planar. Therefore, if $\sigma$ contains 321 or 3412 , then $\mathcal{B}(\sigma)$ is not planar.

On the other hand, Tenner showed [24, Thm. 5.3] that if $\sigma$ avoids both 321 and 3412, then the interval in Bruhat order between the identity and $\sigma$ is isomorphic to the Boolean lattice. In this case, all the edges in the Bruhat 
graph come from covering relations in the Bruhat order, so the Bruhat graph is the edge graph for a cube of dimension $\ell(\sigma)$. (One way to see this is by noting that $\sigma$ avoids 3412 and 4231, so its Bruhat graph has exactly $\ell(\sigma)$ edges at each vertex, and the covering relations in the Boolean lattice already provide $\ell(\sigma)$ edges.) The edge graph of an $n$-dimensional cube is planar if and only if $n \leq 3$. Therefore, $\mathcal{B}(\sigma)$ is planar if and only if $\sigma$ avoids 321 and 3412 and $\ell(\sigma) \leq 3$. Since $\ell(3412)=4$, the condition that $\sigma$ avoid 3412 is encompassed in the condition that $\ell(\sigma) \leq 3$.

We can also prove the above theorem without relying on [24] by directly analyzing the vertices of distance at most 2 from $\sigma$ in $\mathcal{B}(\sigma)$. We show that, if $\sigma$ avoids 321 and 3412, any vertex of distance 2 from $\sigma$ must be connected to exactly 2 vertices of distance 1 from $\sigma$, which suffices to prove the theorem.

The following characterization in terms of the Bruhat graph itself follows immediately from the above proof.

Corollary 3.2. The graph $\mathcal{B}(\sigma)$ is planar if and only if it is a point, a single edge, or the edge graph of a square or a cube.

To state our characterization purely in terms of pattern avoidance, we use the following theorem of Atkinson [1, Thm. 2.3].

Theorem 3.3. Given a permutaton $\pi \in S_{k}$, the set of permutations containing $\pi$ at most $m$ times is characterized by avoiding a finite set of permutations, all of which are in $S_{n}$ for some $n \leq k(m+1)$.

Since the length of a permutation is precisely the number of times it contains the permutation 21, combining Theorems 3.3 and 3.1 produces the following corollary.

Corollary 3.4. The graph $\mathcal{B}(\sigma)$ is planar if and only if $\sigma$ avoids 321 and all permutations $\pi \in S_{m}$ where $m \leq 8$ and $\ell(\pi) \geq 4$.

A computer calculation to reduce the set of permutations given by Corollary 3.4 to a minimal avoidance set produces the following.

Corollary 3.5. The graph $\mathcal{B}(\sigma)$ is planar if and only if $\sigma$ avoids all of the 29 permutations in the following list: 321, 3412, 23451, 23514, 24153, 25134, 31452, 31524, 41253, 51234, 234165, 231564, 231645, 241365, 214563, 214635, 215364, 216345, 314265, 312564, 312645, 412365, 2315476, 2143675, 2143756, 2145376, 2153476, 3125476, 21436587.

Finally, we record the number of permutations whose Bruhat graphs are planar according to length, which follows from [24, Cor. 5.5]:

Corollary 3.6. For any $m \geq 1$, there are 


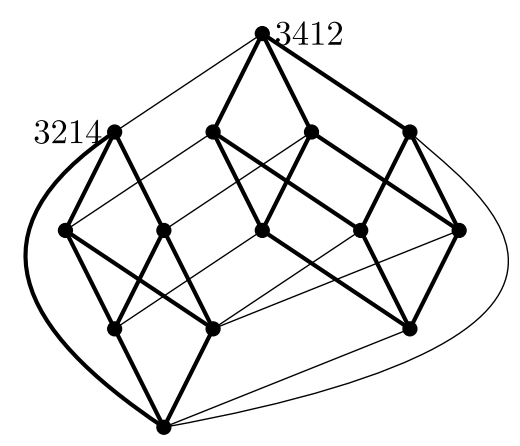

Figure 2: The Bruhat graph $\mathcal{B}(3412)$.

1. 1 permutation in $S_{m}$ of length 0 ,

2. $(m-1)$ permutations in $S_{m}$ of length 1 ,

3. $\frac{(m+1)(m-2)}{2}$ permutations in $S_{m}$ of length 2 , and

4. $\frac{(m+4)(m-1)(m-3)}{6}$ permutations in $S_{m}$ of length 3

which have planar Bruhat graphs.

\section{Toroidal Bruhat graphs}

Now we characterize the permutations whose Bruhat graphs are toroidal.

Theorem 4.1. Let $\sigma$ be a permutation. Then the Bruhat graph $\mathcal{B}(\sigma)$ is toroidal if and only if all three of the following conditions hold:

- $\sigma$ avoids 3412,

- If $\sigma$ contains 321, then $\ell(\sigma)=3$,

- $\ell(\sigma) \leq 4$.

Proof. First we show that the graph $G=\mathcal{B}(3412)$, drawn in Figure 2, is not toroidal. Consider the subgraph $H=\mathcal{B}(3214) \subseteq G$ and the induced subgraph $K \subseteq G$ formed by the vertices not in $H$; these subgraphs are drawn with thicker lines in the figure, with $H$ to the left and $K$ to the right. The subgraph $H$ is isomorphic to the complete bipartite graph $K_{3,3}$, and, in any drawing of $K_{3,3}$ on the torus, each face is homeomorphic to the plane. This is because, if there is a face not homemorphic to the plane, we can take a loop representing a nontrivial element of the fundamental group of this face and, without disturbing the drawing, cut the torus along this loop and cap both resulting holes, resulting in an embedding of $K_{3,3}$ on the sphere, 
violating Theorem 2.4. Furthermore, each vertex in $H$ is connected to at least one vertex of $K$. The subgraph $K$ is isomorphic to the edge graph of a cube, which cannot be drawn on the plane with all the vertices appearing on a single face. (In other words, it is not outerplanar.)

Suppose for contradiction we have a drawing of $G$ on a torus with no edges crossing. If vertices of $K$ are found on two different faces of the drawing of $H$, then there must be a crossing between an edge of $K$ and an edge of $H$. On the other hand, if all the vertices of $K$ are found on a single face $\Phi$ of the drawing of $H$, then recall that (assuming this drawing of $H$ has no crossings), $\Phi$ is homeomorphic to the plane. Since $K$ cannot be drawn on the plane with all the vertices appearing on a single face, there must be a vertex $v \in K$ which is not on the unbounded face of $K$ as drawn in $\Phi$. There is an edge between $v$ and some vertex of $H$, and this edge must cross some edge of $K$. Hence, if $\sigma$ contains $3412, \mathcal{B}(\sigma)$ is not toroidal.

Now we show that, if $\sigma$ contains 321 and $\ell(\sigma) \geq 4$, then $\mathcal{B}(\sigma)$ is not toroidal. We first find a transposition $t$ such that $\ell(\sigma t)<\ell(\sigma)$ and $\sigma t$ also contain 321. Fix indices $i_{1}<i_{2}<i_{3}$ such that $\sigma\left(i_{1}\right)>\sigma\left(i_{2}\right)>\sigma\left(i_{3}\right)$. Since $\ell(\sigma) \geq 4$, there must be an inversion of $\sigma$ involving some index $j \notin\left\{i_{1}, i_{2}, i_{3}\right\}$. If there is such an inversion involving two indices $j_{1}, j_{2} \notin\left\{i_{1}, i_{2}, i_{3}\right\}$, let $t$ be the transposition $t=\left(\begin{array}{ll}j_{1} & j_{2}\end{array}\right)$. Otherwise, we divide first into three cases, where $j<i_{1}$, where $i_{1}<j<i_{3}$, and where $i_{3}<j$. If $j<i_{1}$, let $i \in\left\{i_{1}, i_{2}, i_{3}\right\}$ be the minimum index such that $\sigma(j)>\sigma(i)$ (so that indices $i$ and $j$ form an inversion), and let $t=(j i)$. If $j>i_{3}$ let $i \in\left\{i_{1}, i_{2}, i_{3}\right\}$ be the maximum index such that $\sigma(i)>\sigma(j)$ (so that indices $i$ and $j$ form an inversion), and let $t=(j i)$. If $i_{1}<j<i_{3}$, we need several further subcases. If $j$ and $i_{2}$ form an inversion, then let $t=\left(j i_{2}\right)$. Otherwise, if $j<i_{2}$, then, since $j$ and $i_{2}$ do not form an inversion, $\sigma(j)<\sigma\left(i_{2}\right)<\sigma\left(i_{1}\right)$, so $j$ and $i_{1}$ form an inversion, and we let $t=\left(j i_{1}\right)$. Finally, if $i_{2}<j<i_{3}$ and $\sigma\left(i_{2}\right)<\sigma(j)$, let $t=\left(j i_{3}\right)$. Since $t$ corresponds to an inversion of $\sigma, \ell(\sigma t)<\ell(\sigma)$. In the case where $i_{1}<j<i_{3}$, the permutation $\sigma t$ contains 321 at the indices $t\left(i_{1}\right), t\left(i_{2}\right)$, and $t\left(i_{3}\right)$; in the other cases, the permutation $\sigma t$ contains 321 at the indices $i_{1}$, $i_{2}$, and $i_{3}$.

Given such a transposition $t$, we note that, by Proposition 2.3, $\mathcal{B}(\sigma)$ includes two disjoint subgraphs isomorphic to $\mathcal{B}(321)$, one with the sink at vertex $\sigma$ and the other with the sink at vertex $\sigma t$. (In the case where $\sigma$ and $\sigma t$ contain 321 at the same indices $i_{1}<i_{2}<i_{3}$, the two subgraphs are disjoint since the sets $\left\{\sigma\left(i_{1}\right), \sigma\left(i_{2}\right), \sigma\left(i_{3}\right)\right\}$ and $\left\{\sigma t\left(i_{1}\right), \sigma t\left(i_{2}\right), \sigma t\left(i_{3}\right)\right\}$ are not equal. In the last case where $\sigma$ and $\sigma t$ contain 321 at different indices, the two subgraphs are disjoint since the sets $\left\{\sigma\left(i_{1}\right), \sigma\left(i_{2}\right), \sigma\left(i_{3}\right)\right\}$ and $\left.\sigma t\left(t\left(i_{1}\right)\right), \sigma t\left(t\left(i_{2}\right)\right), \sigma t\left(t\left(i_{3}\right)\right)\right\}$ are the same.) Hence $\mathcal{B}(\sigma)$ contains two disjoint 
subgraphs neither of which are planar. Since $\mathcal{B}(\sigma)$ is connected, a theorem of Battle, Harary, Kodama, and Youngs [4] (see also [18, Thm. 3.5.3]) now implies that $\mathcal{B}(\sigma)$ cannot be toroidal.

If $\sigma$ contains 321 and $\ell(\sigma)=3$, then $\mathcal{B}(\sigma)$ is isomorphic to $\mathcal{B}(321)$, which is $K_{3,3}$. This graph is well known to be toroidal.

Finally, due to a theorem of Tenner [24] previously mentioned in the proof of Theorem 3.1, if $\sigma$ avoids both 321 and 3412, then $\mathcal{B}(\sigma)$ is the edge graph of a cube of dimension $\ell(\sigma)$. The edge graph of the 4-dimensional cube is toroidal, but the edge graph of the 5 -dimensional cube is not.

As before, we can give this characterization in terms of the Bruhat graph.

Corollary 4.2. The graph $\mathcal{B}(\sigma)$ is toroidal if and only if it is a point, a single edge, the edge graph of a square, a cube, or a 4-cube, or the complete bipartite graph $K_{3,3}$.

Using Theorem 3.3, we see the following.

Corollary 4.3. The graph $\mathcal{B}(\sigma)$ is toroidal if and only if $\sigma$ avoids all permutations $\pi \in S_{m}$ where $m \leq 10$ and $\mathcal{B}(\pi)$ is not toroidal.

A computer calculation produces the following explicit minimal list of 92 permutations that must be avoided.

Corollary 4.4. The graph $\mathcal{B}(\sigma)$ is toroidal if and only if $\sigma$ avoids all of the following permutations: 3412, 4321, 4213, 4312, 3241, 4231, 4132, 3421, 2431, 32154, 21543, 234561, 234615, 235164, 236145, 241563, 241635, 251364, 261345, 314562, 314625, 315264, 316245, 412563, 412635, 512364, 612345, 2145673, 2145736, 2146375, 2147356, 2153674, 2153746, 2163475, 2173456, 2315674, 2315746, 2316475, 2317456, 3125674, 3125746, 3126475, 3127456, 2341675, 2413675, 3142675, 4123675, 2341756, 2413756, 3142756, 4123756, 2345176, 2351476, 2415376, 2513476, 3145276, 3152476, 4125376, 5123476, 23416587, 24136587, 31426587, 41236587, 21456387, 21463587, 21536487, 21634587, 21436785, 21436857, 21437586, 21438567, 23156487, 23164587, 31256487, 31264587, 23154786, 23154867, 31254786, 31254867, 21453786, 21453867, 21534786, 21534867, 231547698, 312547698, 214537698, 215347698, 214367598, 214375698, 214365897, 214365978, 21436587 A9.

The number of permutations with toroidal Bruhat graphs, enumerated by length, is as follows.

Corollary 4.5. For an $m \geq 1$, there are

1. 1 permutation of length 0 , 
2. $(m-1)$ permutations of length 1 ,

3. $\frac{(m+1)(m-2)}{2}$ permutations of length 2,

4. $\frac{(m+4)(m-1)(m-3)}{6}+(m-2)$ permutations of length 3, and

5. $\frac{(m+1)(m-4)\left(m^{2}+5 m-18\right)}{24}$ permutations of length 4

which have toroidal Bruhat graphs.

Proof. A permutation $\sigma \in S_{m}$ which contains 321 and has length 3 must be a transposition of the form $(i i+2)$ for some $i$ with $1 \leq i \leq m-2$. The rest of the enumeration follows from [24, Cor 5.5], as in the case of planar Bruhat graphs.

\section{Acknowledgements}

Most of this work was the result of a summer undergraduate research project at St. Olaf College mentored by the second author. We thank St. Olaf College for the financial support that made this work possible. The first part of the proof of Theorem 4.1, showing that the Bruhat graph of 3412 is not toroidal, is due to Michael Eldredge. We thank him for allowing us to include it in this paper. We also thank Mark Laidlaw for tracking down a copy of [14].

\section{References}

[1] M. D. Atkinson, Restricted permutations, Discrete Math. 195 (1999), 27-38. MR1663866

[2] M. D. Atkinson, M. M. Murphy, and N. Ruškuc, Partially well-ordered closed sets of permutations, Order 19 (2002), 101-113. MR1922032

[3] M. D. Atkinson, M. M. Murphy, and N. Ruškuc, Pattern avoidance classes and subpermutations, Electron. J. Combin. 12 (2005), Research Paper 60, 18 pp. (electronic). MR2180797

[4] J. Battle, F. Harary, Y. Kodama, and J. W. T. Youngs, Additivity of the genus of a graph, Bull. Amer. Math. Soc. 68 (1962), 565-568. MR0155313

[5] S. Billey and T. Braden, Lower bounds for Kazhdan-Lusztig polynomials from patterns, Transform. Groups 8 (2003), 321-332. MR2015254

[6] S. Billey and B. Jones, Embedded factor patterns for Deodhar elements in Kazhdan-Lusztig theory, Ann. Comb. 11 (2007), 285-333. MR2376108 
[7] S. Billey and B. Pawlowski, Permutation patterns, Stanley symmetric functions and generalized Specht modules, J. Combin. Theory, Ser. A 127 (2014), 85-120. MR3252657

[8] S. Billey and A. Postnikov, Smoothness of Schubert varieties via patterns in root subsystems, Adv. in Appl. Math. 34 (2005), 447-466. MR2123545

[9] S. Billey and J. Weed, Appendix A: a purely pattern avoidance characterization to A. Woo, Permutations with Kazhdan-Lusztig polynomial $P_{i d, w}(q)=1+q^{h}$, Electron. J. Combin. 16 (2009), no. 2, Research Paper 10, 32 pp. (electronic). MR2515773

[10] A. Björner and F. Brenti, Combinatorics of Coxeter groups, Graduate Texts in Mathematics 231, Springer, New York, 2005. MR2133266

[11] M. Bóna, Combinatorics of Permutations, Discrete Mathematics and its Applications, Chapman \& Hall/CRC, Boca Raton, FL, 2004. MR2078910

[12] F. Brenti, The intersection cohomology of Schubert varieties is a combinatorial invariant, Europ. J. Combin. 25 (2004), 1151-1167. MR2095476

[13] J. Carrell, The Bruhat graph of a Coxeter group, a conjecture of Deodhar, and rational smoothness of Schubert varieties, in: Algebraic groups and their generalizations: classical methods (University Park, PA, 1991), Proc. Sympos. Pure Math., 56, Part 1, Amer. Math. Soc., Providence, RI, 1994, pp. 53-61. MR1278700

[14] J. Chambers, Hunting for Torus Obstructions, M.Sc. Thesis, Department of Computer Science, University of Victoria, 2002.

[15] E. Delanoy, Combinatorial invariance of Kazhdan-Lusztig polynomials on intervals starting from the identity, J. Algebraic Combin. 24 (2006), 437-463. MR2266379

[16] M. J. Dyer, On minimal lengths of expressions of Coxeter group elements as products of reflections, Proc. Amer. Math. Soc. 129 (2001), 2591-2595. MR1838781

[17] F. du Cloux, Rigidity of Schubert closures and invariance of KazhdanLusztig polynomials, Adv. in Math. 180 (2003), 146-175. MR2019220

[18] J. L. Gross and T. W. Tucker, Topological Graph Theory, WileyInterscience Series in Discrete Mathematics and Optimization, John Wiley \& Sons, Inc., New York, NY, 1987. MR0898434 
[19] V. Lakshmibai and B. Sandhya, Criterion for smoothness of Schubert varieties in $S L(n) / B$, Proc. Indian Acad. Sci. Math. Sci. 100 (1990), 45-52. MR1051089

[20] V. R. Pratt, Computing permutations with double-ended queues, parallel stacks and parallel queues, Proc. ACM Symp. Theory of Computing 5 (1973), 268-277 MR0489115

[21] N. Robertson and P. D. Seymour, Graph minors. VIII. A Kuratowski theorem for general surfaces, J. Combin. Theory Ser. B 48 (1990), 255288. MR1046758

[22] N. Robertson and P. D. Seymour, Graph minors. XX. Wagner's conjecture, J. Combin. Theory Ser. B 92 (2004), 325-357. MR2099147

[23] D. A. Spielman and M. Bóna, An infinite antichain of permutations, Electron. J. Combin. 7 (2000), Note 2, 4 pp. (electronic). MR1741337

[24] B. E. Tenner, Pattern avoidance and the Bruhat order, J. Combin. Theory Ser. A 114 (2007), 888-905. MR2333139

[25] B. E. Tenner, Repetition in reduced decompositions, Adv. in Appl. Math. 49 (1), 1-14. MR2925922

Christopher Conklin

School of Physics \& Astronomy

University OF Minnesota

116 Church St. SE

MinNEAPOLIS, MN 55455

USA

E-mail address: cconklin@me.com

Alexander Woo

Department of Mathematics

UNIVERSITY OF IDAHO

875 Perimeter Drive, MS 1103

Moscow, ID 83844-1103

Russia

E-mail address: awoo@uidaho.edu

Received 3 October 2013 